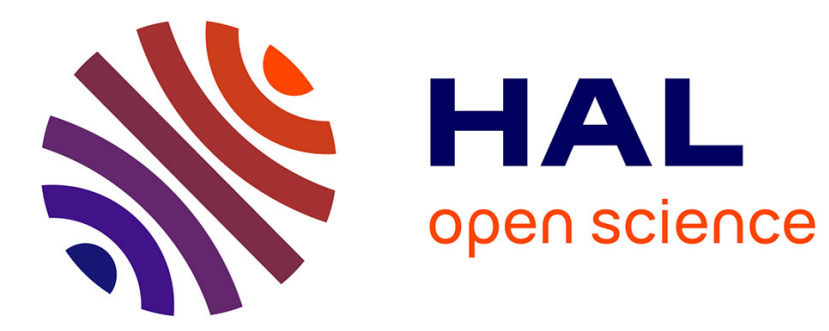

\title{
Wave Farm Flicker Severity: Comparative Analysis and Solutions
}

\author{
Thibaut Kovaltchouk, Sara Armstrong, Anne Blavette, Hamid Ben Ahmed, \\ Bernard Multon
}

\section{- To cite this version:}

Thibaut Kovaltchouk, Sara Armstrong, Anne Blavette, Hamid Ben Ahmed, Bernard Multon. Wave Farm Flicker Severity: Comparative Analysis and Solutions. Renewable Energy, 2016, 91, pp.32-39. 10.1016/j.renene.2016.01.034 . hal-01265905

\section{HAL Id: hal-01265905 \\ https://hal.science/hal-01265905}

Submitted on 1 Feb 2016

HAL is a multi-disciplinary open access archive for the deposit and dissemination of scientific research documents, whether they are published or not. The documents may come from teaching and research institutions in France or abroad, or from public or private research centers.
L'archive ouverte pluridisciplinaire HAL, est destinée au dépôt et à la diffusion de documents scientifiques de niveau recherche, publiés ou non, émanant des établissements d'enseignement et de recherche français ou étrangers, des laboratoires publics ou privés.

\section{(ㅇ)(1) $\$$}

Distributed under a Creative Commons Attribution - NonCommercial - NoDerivatives| 4.0 


\title{
Wave Farm Flicker Severity: Comparative Analysis and Solutions
}

\author{
Thibaut Kovaltchouk ${ }^{\mathrm{a}, *}$, Sara Armstrong ${ }^{\mathrm{b}}$, Anne Blavette ${ }^{\mathrm{a}}$, Hamid Ben Ahmed ${ }^{\mathrm{a}}$, Bernard Multon ${ }^{\mathrm{a}}$ \\ ${ }^{a}$ SATIE CNRS UMR8029, ENS Rennes, UEB, Av. Robert Schuman, Bruz, France \\ ${ }^{b}$ Marine and Renewable Energy Ireland, University College Cork, Cork, Ireland
}

\begin{abstract}
This paper proposes a flicker severity study for Wave Energy Converter farms. The flicker severity is introduced and the reason why it is an important constraint for a wave farm is explained. A new representation called intrinsic flicker severity is introduced which describes the flicker severity independently of the grid. The influence of device type, its control and the sea-state on average production, flicker severity and on the ratio between flicker and production are studied with three types of devices: an Oscillating Water Column and two Direct Wave Energy Converters (two point absorbers: a Heaving Buoy and the SEAREV). The influence of the size and the placement of each unit in the wave farm is presented with a farm-unit flicker ratio, compared with the square-root of unit hypothesis (noise behavior), as a function of wave direction by taking into account wave direction dispersion. Finally, solutions are presented to reduce the flicker produced to comply with grid code requirements in order to allow grid integration of wave farms.
\end{abstract}

Keywords: Wave Energy, Wave Farm, Power Quality, Flicker, Oscilating Water Colum, Electrical Energy Storage

\section{Introduction}

Integration to the grid is one of the keys to commercializing Wave Energy Converters (WECs). However, some WEC technologies produce power that fluctuates at the rate of ocean waves. Some of them have intrinsic energy storage from the pneumatic or hydraulic chain, but can still have other issues, like efficiency and reliability.

When the produced power fluctuates at frequencies between $5 \mathrm{mHz}$ and $33 \mathrm{~Hz}$, the induced rms-voltage fluctuations can cause power quality problems at the grid connection point. WECs have been identified as particularly susceptible to induce flicker [1-4]. The combination of the weak grid (which could be the case with an island or a near-shore distribution grid) and production fluctuations can cause flicker non-compliance, relative to the grid code requirements. In order to analyze this issue, a new representation called intrinsic flicker is proposed in order to represent the results under several conditions; such as grid characteristics and reactive power injection.

Wave farm flicker severity depends on several influences; device type, different controls, sea-states, sizes of farms and architecture of farm. A comparison of different device types is proposed here: oscillating water column (OWC), heaving buoy, and the SEAREV (see Fig. 1). Some studies has already been

*thibaut.kovaltchouk@ens-rennes.fr (C) 2016. 다)(앙 This manuscript version is made available under the $\mathrm{CC}$ BY-NC-ND 4.0 license.

Author version of the following accepted manuscript:

Thibaut Kovaltchouk, Sara Armstrong, Anne Blavette, Hamid Ben Ahmed, Bernard Multon, Wave farm flicker severity: Comparative analysis and solutions, Renewable Energy, Volume 91, June 2016, Pages 32-39, ISSN 09601481, http://dx.doi.org/10.1016/j.renene.2016.01.034. made on the flicker severity, but are often limited: [3][5] are interested in one device type (OWC) and one control strategy for one sea-state for a 22 and 6 units wave farm respectively, with investigation of only two wave directions. In [6], one type of device (OWC) is investigated with two different controls for several sea-states and different wave farm sizes but does not investigate architecture and wave direction. Reference [7] discusses one device type (Wavebob) and one control with just one unit.

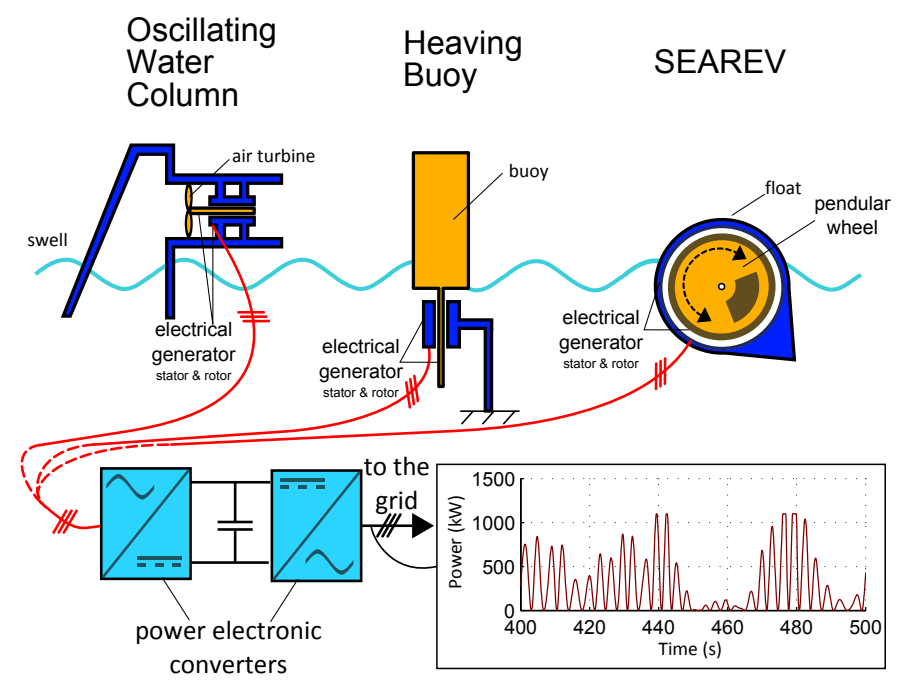

Figure 1: Three types of Wave Energy Converter with a strong pulsating power production used for this study: an Oscillating Water Column, a Heaving Buoy and the SEAREV.

This work will focus on relative small farms (5 to $20 \mathrm{MW}$ ) connected to a medium voltage distribution grid (1 to $50 \mathrm{kV})$. 
Section 2 introduces the flicker constraint, and proposes a definition for an intrinsic flicker severity, that allows a generalization of the results for all grid characteristics at the PCC (Point of Common Coupling) and grid codes. Section 3 presents the model used for the three different devices and their control. Then, in Section 4 the different influences are presented with flicker-production ratio and farm-unit flicker ratio in order to compare different cases. The classical hypothesis used in wind energy [8] that states this ratio must be near $\sqrt{N}$ (that is that each unit is considered as an independent noise source) will be compared to the results. Finally, Section 5 proposes solutions to reduce flicker to allow grid integration of wave farms.

\section{Flicker and Flickermeter}

\subsection{Flicker Definition and Standards}

To enable grid integration, energy producers must meet some constraints on the quality of injected energy. The limitation of voltage fluctuations (flicker) is a critical constraint for WECs. Power-line flicker is a visible change in brightness of a light source due to rapid fluctuations in the power supply voltage. These fluctuations are caused by variations in either active or reactive power to the network [9]. Beyond a certain amplitude, these rapid fluctuations (in a range from $5 \mathrm{mHz}$ to $33 \mathrm{~Hz}$ ) may cause humans to suffer from fatigue, irritability and epilepsy but can also cause premature aging of electrical devices [10]. So these fluctuations are constrained by flicker standards to keep them limited (see Fig. 2).

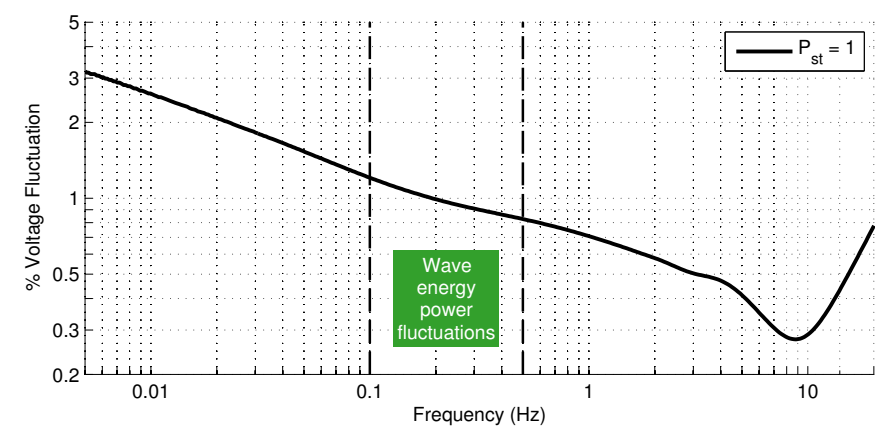

Figure 2: Flicker constraint: $P_{s t}=1$ curve for regular rectangular voltage changes according to the IEC 61000-4-15 Standard. The zone above the curve corresponds to irritation for the consumer. Wave power fluctuations typically fall between 0.1 and $0.5 \mathrm{~Hz}$.

Two flicker severities are typically used in grid codes:

- The short-term flicker severity $P_{s t}$ is measured over a 10 minute period,

- The long-term flicker severity $P_{l t}$ is measured over 2 hours.

Flicker measurement with a flickermeter is defined in the IEC 61000-4-15 Standard [9]. Three blocks constitutes a flickermeter:

- The first process is a demodulation process that involves squaring and filtering the input voltage profile in order to compute rms-voltage waveform.
- This waveform then goes through the simulation filters which have a maximum value at $8.8 \mathrm{~Hz}$ that simulates the lamp to eye response, whilst a squaring operation and a sliding mean filter simulate the non-linear memory process in the eye and brain.

- The extent to which flicker is annoying to the observer depends on its level and its rate of occurrence. This is carried-out by a distribution which relates to the proportion of time each particular level of flicker is exceeded. After 10 minutes of data accumulation, key levels are taken from this distribution to compute the short-term flicker severity $P_{s t}$.

A flickermeter installed in Matlab is used [11]. Further details on the use of this flickermeter can be found in [12]. The short-term flicker $P_{s t}(\Delta V(t) / V)$ is used in the following as a function of a 10-minutes temporal profile $\Delta V(t) / V$ and similarly the long-term flicker $P_{l t}(\Delta V(t) / V)$ as a function of a 2hours temporal profile $\Delta V(t) / V$.

The long term flicker severity $P_{l t}$ is simply calculated with twelve consecutives values of the short term flicker severity $P_{s t i}$ :

$$
P_{l t}=\left(\frac{1}{12} \sum_{i=1}^{12} P_{s t i}^{3}\right)^{1 / 3}
$$

By definition, a flicker severity equal to 1 for the $P_{s t}$ corresponds to the acceptable limits that the electricity distribution must provide to its customers and the limit for $P_{l t}$ is 0.8 .

\subsection{Flicker Emission limits}

To ensure these levels, the distributor system operator requires consumers and producers to limit their individual flicker contribution to lower levels (due to pollution aggregation).

$$
\begin{aligned}
P_{s t} & \leq P_{\text {st max }} \\
P_{l t} & \leq P_{l t \text { max }}
\end{aligned}
$$

Table 1 gives the individual flicker severity limits according to the IEC standard[13] and some grid codes. These limits consider only the effect of the producer (or consumer) at its PCC, because the producer (or consumer) can only be responsible for maintaining his emissions. The utility is responsible for the overall control of disturbance within the electrical grid, and we want to consider here only the wave farm responsibility.

The wave elevation considered in this study is polychromatic and is described with different sea-states (each one described by its spectrum defined with its significant height and its peak period). If this phenomenon is stationary for a duration of around two hours, a relationship exists between short and long term flicker severities:

$$
P_{l t} \approx P_{s t}
$$

The long-term severity is more stringent and hence will be used in this study. Short-term severity is used instead when there are non-regular events, like grid connection/disconnection stages, while the long-term flicker can take into account a more typical flicker injection. 
Table 1: Individual flicker severities limits (Medium Voltage)

\begin{tabular}{lcc}
\hline & $P_{\text {st } \max }$ & $P_{l t \max }$ \\
\hline IEC 61000-3-7[13] & $0.35^{1}$ & $0.25^{1}$ \\
France [14] & 0.35 & 0.25 \\
Ireland (Wind/wave) [15] & 0.35 & 0.35 \\
United-Kingdom [16] & $0.50^{1}$ & $\mathrm{~N} / \mathrm{A}$ \\
Denmark (Wind)[17] & $0.30^{4}$ & $0.50^{2} / 0.35^{3} / 0.20^{4}$ \\
\hline
\end{tabular}

${ }^{1}$ Basic limits, further explanations in 5.1

${ }^{2} U_{n} \leq 35 \mathrm{kV}$

${ }^{3} 35 \mathrm{kV}<U_{n} \leq 100 \mathrm{kV}$

${ }^{4} U_{n}>100 \mathrm{kV}$

\subsection{Voltage Fluctuations Computation}

The voltage fluctuation $\Delta V(t)$ due to a WEC farm at the point of connection is calculated by following this conventional approximated formula, sufficiently accurate with respect to flicker:

$$
\begin{aligned}
\frac{\Delta V(t)}{V} & =\frac{P_{\text {Grid }}(t) \cdot \cos (\Psi)+Q_{\text {Grid }}(t) \cdot \sin (\Psi)}{S_{k}} \\
\frac{\Delta V(t)}{V} & =P_{\text {grid }}(t) \frac{\cos (\Psi)(1+\tan (\phi) \tan (\Psi))}{S_{k}}
\end{aligned}
$$

with $P_{\text {grid }}(t)$ and $Q_{\text {grid }}(t)$ respectively the active and reactive power provided by the WEC farm (generator convention, i.e. positive value when it produces power), $\phi=$ $\arctan \left(Q_{\text {grid }}(t) / P_{\text {grid }}(t)\right)$ the angle between the current and the voltage, $\Psi=\arctan (X / R)$ the grid impedance angle and $S_{k}$ the short-circuit apparent power at the PCC. This simplification is used by lot of flicker studies and some standards [8, 13, 18, 19] because the voltage fluctuations that corresponds to the flicker constraints are relatively small (around $1 \%$ for the wave frequencies, cf. Fig 2). Of course, this formula indicates only the voltage fluctuations attributable to the wave farm, and so, its participation to the flicker severity at this point.

\subsection{Intrinsic Flicker Severity}

In the following, the long-term flicker severity $P_{l t}(x(t))$ is considered as a function of any temporal profile $x(t)$. Let's notice that this function is homogeneous, which means that, for the same shape of voltage fluctuations (in particular same period), the severities are proportional to the amplitude of voltage fluctuations. So the flicker severity due to the farm can be rewritten as the product of two terms:

$$
\begin{aligned}
P_{l t}\left(\frac{\Delta V(t)}{V}\right) & =P_{l t}\left(P_{\text {grid }}(t)\right) \frac{\cos (\Psi)|1+\tan (\phi) \tan (\Psi)|}{S_{k}} \\
P_{l t}\left(\frac{\Delta V(t)}{V}\right) & =P_{l t P} \frac{\cos (\Psi)|1+\tan (\phi) \tan (\Psi)|}{S_{k}}
\end{aligned}
$$

with $|1+\tan (\phi) \tan (\Psi)|$ the absolute value of $(1+\tan (\phi) \tan (\Psi))$.

Therefore, one term depends only on the power profile $\left(P_{l t P}=P_{l t}\left(P_{\text {grid }}(t)\right)\right.$, in $\left.M V A\right)$, that will be referred to as the intrinsic flicker severity from hereon in, and the other depends on the grid characteristics $\left(S_{k}\right.$ and $\Psi$ ) and reactive power injection policy $(\tan (\phi))$ often limited by the grid code. This intrinsic flicker has no physical meaning and is just a mathematical intermediate result: the output of the $P_{l t}()$ function that takes as an input a given power temporal profile. Intrinsic flicker has a similar purpose to the flicker coefficient used for wind turbines [8]. However, the latter is based on normalized flicker severity independent of the short-circuit apparent power $S_{k}$ only. In other words, it still depends on the grid characteristics as it depends on the grid impedance angle. On the contrary, our flicker coefficient is independent of both the short-circuit apparent power $S_{k}$ and of the grid impedance angle. Hence, it is a measure of a device intrinsic flicker potential which is completely independent of the grid characteristics. Consequently, using this index should greatly facilitate the estimation of flicker generated by a wave farm connected to any type of grid, regardless of its characteristics.

As seen in section 2.2, the flicker severity $P_{l t}$ is limited. Subsequently, there is a constraint on the power fluctuations (measured by the intrinsic flicker severity $P_{l t P}$ ) produced by the farm:

$$
P_{l t P \max }=P_{l t \max } \frac{S_{k}}{\cos (\Psi)|1+\tan (\phi) \tan (\Psi)|}
$$

So the maximum value allowed for the intrinsic flicker severity $P_{l t P \text { max }}$, which is derived from the maximum flicker limit $P_{l t \text { max }}$ enforced by grid operators, depends on the grid characteristics. According to [20], typical short circuit power in a medium voltage grid is between 10 and 2500 MVA and typical X/R ratio is around 1.5. The standard for wind turbines [8] recommends to verify the flicker severity for $\mathrm{X} / \mathrm{R}$ ratio between 0.58 and 11.4 (angle of the grid impedance: $30^{\circ}, 50^{\circ}, 70^{\circ}$ and $85^{\circ}$.

Fig. 3 shows the ratio between the maximum allowed intrinsic flicker severity $P_{l t P \max }$ and the fault level $S_{k}$ for X/R ratio between 0.5 and 3.5 and $\mathrm{Q} / \mathrm{P}$ ratio between 0 and -1.2 , with the hypothesis that the maximum allowed flicker severity equals $P_{l t \max }=0.25$. Evidently, the value for the allowed flicker severity becomes very important when the $\mathrm{Q} / \mathrm{P}$ ratio is near $-\mathrm{R} / \mathrm{X}$ ratio.

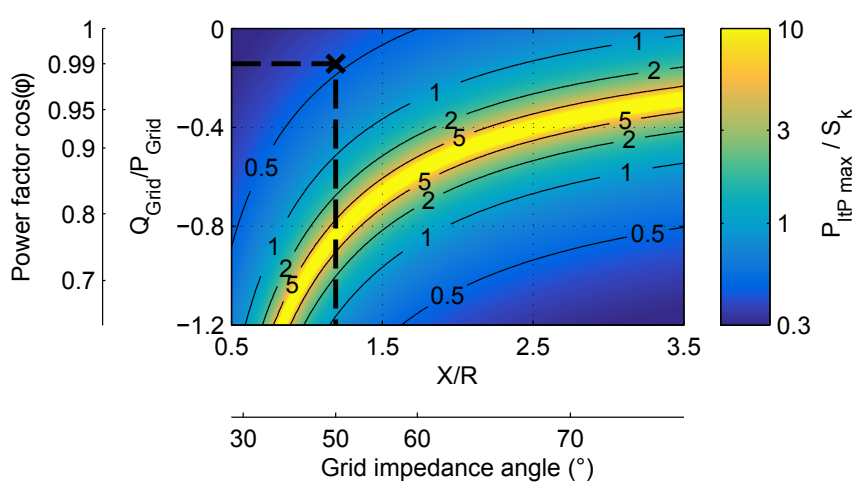

Figure 3: Ratio between the maximum allowed value for the intrinsic longterm flicker severity $P_{l t P \max }$ and the short-circuit apparent power of the grid $S_{k}$ as a function of $\mathrm{X} / \mathrm{R}$ ratio and $\mathrm{Q} / \mathrm{P}$ ratio (equation (9))

The major advantage of the intrinsic flicker severity $\left(P_{l t P}\right)$ indicator is to allow the computation of the flicker severity in all 
Table 2: Devices studied with their controls

\begin{tabular}{|c|c|c|c|}
\hline Device & Oscillating Water Column (OWC) & Heaving Buoy (HB) & SEAREV \\
\hline Principle & $\begin{array}{l}\text { Air displaced by the water in a chamber } \\
\text { and pushed back and forth past a Wells } \\
\text { turbine connected to an electrical gener- } \\
\text { ator }\end{array}$ & $\begin{array}{l}\text { The rise and fall of the waves moves the } \\
\text { buoy connected to a linear electrical gen- } \\
\text { erator }\end{array}$ & $\begin{array}{l}\text { Floating device enclosing a heavy hori- } \\
\text { zontal axis wheel connected to an elec- } \\
\text { trical generator }\end{array}$ \\
\hline Dimensions & $\mathrm{W}=8 \mathrm{~m} ; \mathrm{L}=8 \mathrm{~m} ; \mathrm{H}=10 \mathrm{~m}$ & $D=10 m ; H=15 m$ & $\mathrm{~W}=30 \mathrm{~m} ; \mathrm{D}=10 \mathrm{~m}$ \\
\hline Controls & $\begin{array}{l}\text { Constant or optimal speed reference } \\
\text { (constant speed optimized for each sea- } \\
\text { state) }\end{array}$ & $\begin{array}{l}\text { Passive or reactive with force level- } \\
\text { ing }(1 \mathrm{MN}) \text { and power leveling }(1 \mathrm{MW}) \text {, } \\
\text { control optimized for each sea-state }\end{array}$ & $\begin{array}{l}\text { Passive with power leveling (1.1 MW), } \\
\text { control optimized for one sea-state }\end{array}$ \\
\hline References & Cashman et al.[21] & Kovaltchouk et al.[22] & Aubry et al.[23] \\
\hline
\end{tabular}

possible cases (depending on grid characteristics and grid policy). For example, (9) or Fig.3 can be used to determine that for grid characteristics of $S_{k}=40 \mathrm{MVA}$, and $\Psi=50^{\circ}$, and reactive power consumption that corresponds to $\phi=-\arccos (0.99)$, we have: $P_{l t P \text { max }}=0.47 \cdot S_{k}=19 \mathrm{MVA}$.

\section{Model and Hypotheses}

\subsection{Device Types}

Three WEC technologies are studied in this paper (see Fig.1): an oscillating water column (OWC) and two Direct Wave Energy Converters (DWEC); a heaving buoy and the SEAREV). These are fully presented in Table 2. The models of the OWC, the heaving buoy and the SEAREV used in this paper have been described in detail in [21], [22], [23] respectively.

\subsection{Farm Model}

Similarly to [24] and [5], the model of the aggregate production of a farm is done using temporal delay of a power profile production in order to find the production of each unit, so hydrodynamic interactions are not considered. These time delays are computed using the group velocity $\overrightarrow{v_{g}}$ and the distance between the two units $\overrightarrow{O_{i} O_{j}}$ :

$$
\Delta T_{i j}=\frac{\overrightarrow{O_{i} O_{j}} \cdot \overrightarrow{v_{g}}}{{\overrightarrow{v_{g}}}^{2}}
$$

Here we suppose that the group velocity $\overrightarrow{v_{g}}$ depends of the sea-state through the peak period $T_{p}$ :

$$
\left\|\overrightarrow{v_{g}}\right\|=\frac{g T_{p}}{4 \pi}
$$

The minimum distance between the units is 200 meters, as recommended by [25] to limit negative effect on the wave global efficiency. We can suppose that the effects of these interactions are relatively low concerning flicker severities.

For each farm size (7, 13 and 19 units), two different architectures are compared: 2 lines or the most compact configuration possible (see Fig. 4, bottom row).
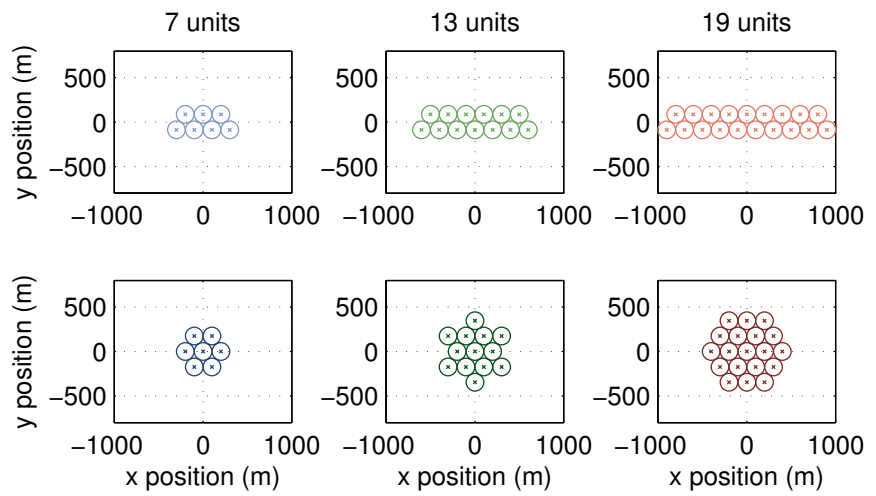

Figure 4: The six architectures studied with three different numbers of units: 7,13 and 19 units. The units positions are represented by cross. The diameter of the circles correspond to $200 \mathrm{~m}$.

\section{Results}

\subsection{Influence of Device Type and Control}

Fig. 5 shows the average production, the intrinsic flicker and the ratio between these parameters for 5 sea-states (significant height: $3 \mathrm{~m}$, peak period: 7, 9, 10, 12 and $14 \mathrm{~s}$ ) and for three device types and five control modes (results for a single WEC). These are the OWC with a constant or variable speed control, heaving buoy with a passive and reactive control, and the SEAREV with a passive control. The choice for the control parameters was done in all these cases to maximize average electrical production (as described in [26]). The values of the bar graph correspond to the sea-state $\left(H_{s}=3 \mathrm{~m}, T_{p}=10 \mathrm{~s}\right)$ and the incertitude bars correspond to the other sea-states.

For a device and its associated control, the ratio between the intrinsic flicker and the production seems to have less variation. Globally, all the values of ratio are between 50 and 250 VA/W. The two OWC ratios are similar, despite the possibility to use the machine and turbine inertia with the variable speed control to smooth power fluctuations. Indeed, the goal of this control was to maximize power control, and thus has no better flickerpower ratio. By contrast, the heaving buoy has very different ratios depending on the control. The two Direct Wave Energy Converters with a passive control have similar values of ratio.

\subsection{Influence of Sea-State}

Fig. 6 shows the average production, the intrinsic flicker and the ratio between these values, for varying levels of peak pe- 

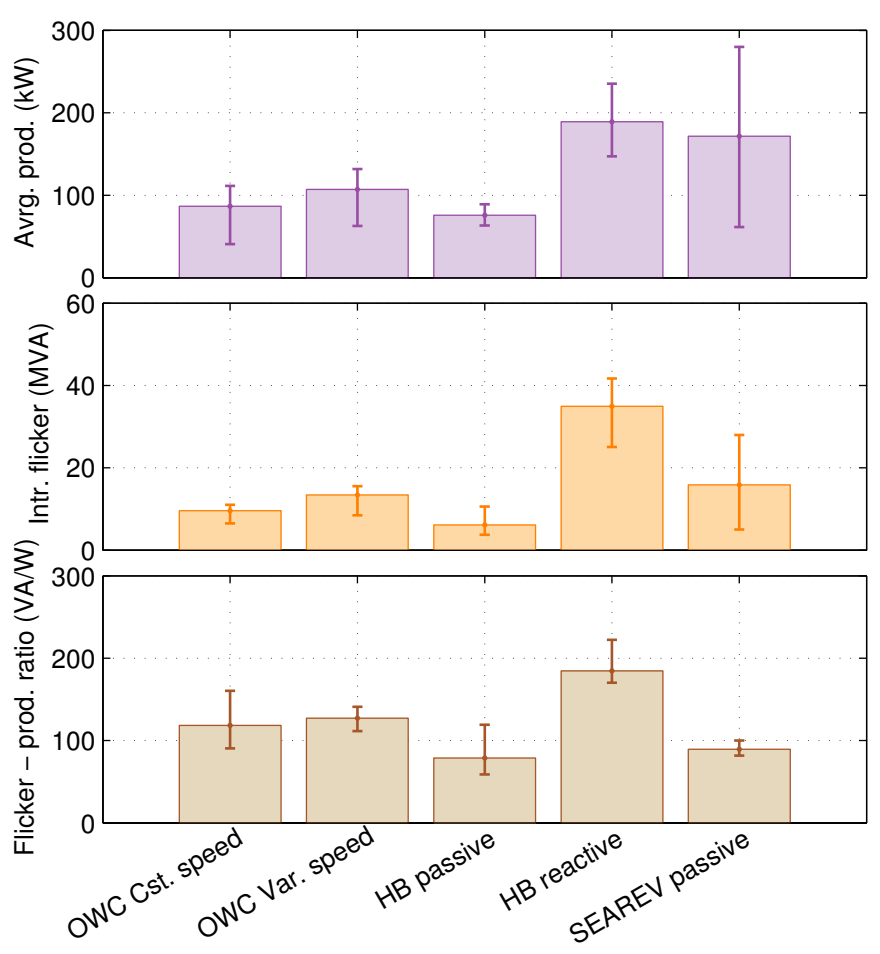

Figure 5: Average power production, intrinsic flicker and flicker-power ratio for the five cases considered here: Oscillating Water Column with constant and variable speed reference, Heaving Buoy with passive or reactive control and the SEAREV with passive control.

riod. Results from simulations of a single WEC are shown here and are used to optimize the control parameters. These are normalized by the value for the sea-states with a significant height of $3 \mathrm{~m}$ and the peak period of $10 \mathrm{~s}$.

It can be seen that the correlation between flicker severity and average production is important. This is logical because power fluctuations and average power are directly linked. So, because the different WEC types considered in this study have different average productions as a function of sea-state, the maximum value of flicker can correspond to very different sea-states.

It can also be seen that, globally, the flicker-production ratio decreases with an increase in the peak-period, that is consistent with the flicker severity that is stronger for bigger frequency (see Fig. 2). This function also depends on the device type and its control, so this could make very difficult to standardize flicker verification for wave energy devices with only few representative sea-states. This kind of verification are easier for wind turbines with just few different wind speeds used in some standards [8].

\subsection{Influence of the Number of Units and Farm Architecture}

In this section, only one production profile from a heaving buoy with a reactive control is used, given that the results from the other case studies are very similar. Further information on the hydro-mechanical and PTO models used to run this simulation can be found in [26].

Fig. 7 shows the intrinsic flicker severity of a farm normalized by the intrinsic flicker severity of one unit, that is
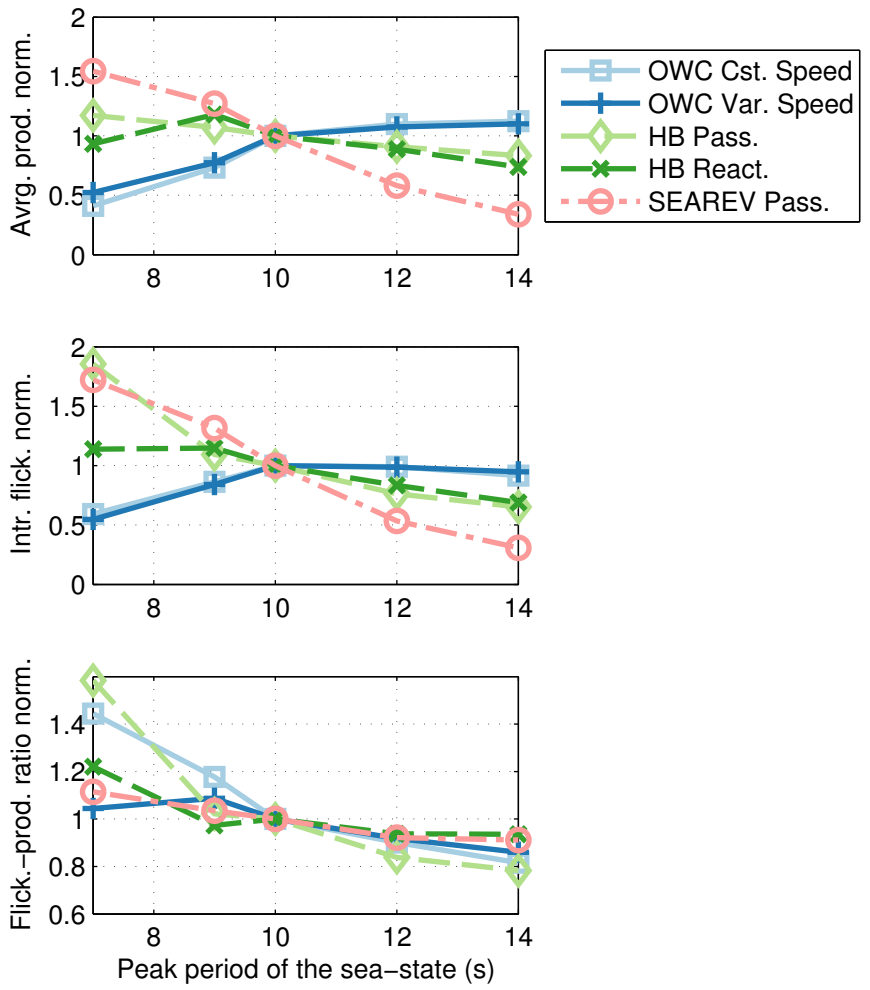

Figure 6: Average power production, intrinsic flicker and flicker-power ratio as a function of the peak period of the sea-state (constant significant wave height of 3 meters) for the five cases considered here: Oscillating Water Column with constant and variable speed reference, Heaving Buoy with passive or reactive control and the SEAREV with passive control. All the values are normalized in relation to the value for the sea-state $\left(H_{s}=3 \mathrm{~m}, T_{p}=10 \mathrm{~s}\right)$.

a farm-unit flicker ratio, as a function of the wave direction $\left(0^{\circ}\right.$ correspond to the $\mathrm{x}$-axis of figure 4$)$.

The classical hypothesis is to assume that this value equals the square-root of the number of units in the farm $\sqrt{N}$, because power fluctuations are supposed to be statically independent. It can be seen that, for some directions, the farm-unit flicker ratio is bigger than expected with this hypothesis; this case corresponds to several units having the same production because there are aligned front to the waves.

The fact that units separated with at least $200 \mathrm{~m}$ have exactly the same production profile is not realistic for a number of reasons: wave dispersion, WEC interactions, WEC movements within its mooring limits, and so on. Here, we present a second scenario by taking into account the fact that the waves are multi-directional: the direction spreading is modeled using a cosine 2 s distribution (proportional to $\cos \left(\frac{\theta-\bar{\theta}}{2}\right)^{2 s}$ ) [27]. The value, $s=10$, corresponds to a narrow spread. Of course, this value, like others that defines a sea-state, have a high correlation with the wind speed [28].

So, the results shown in Fig. 7 are smoothed using this cosine 2 s function as a kernel: these results are shown in Fig. 8. This choice to smooth our results according to wave dispersion is not standard but seems to give more realistic results with the use of only power production profiles. Future work will focus on the comparison in terms of flicker estimation of this model- 

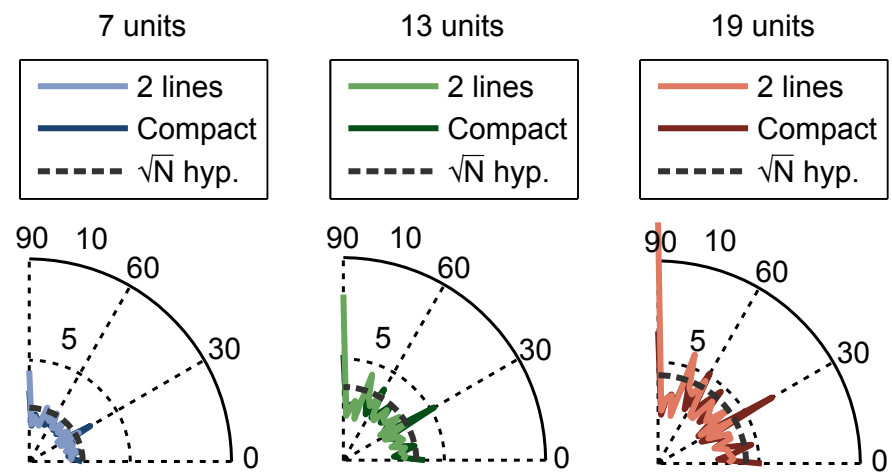

Figure 7: Farm-unit flicker ratio (ratio of the wave farm flicker severity to the unit flicker severity) as a function of the wave direction (monodirectional waves) with 2 type of architecture and 3 different numbers of devices.

ing approach to a more detailed model based on hydrodynamic equations (computation of wave elevation time-series at each unit).

It can be noticed that the placement of the devices still has an importance, and placing units aligned front to the waves main direction must be avoided to reduce the flicker severity.

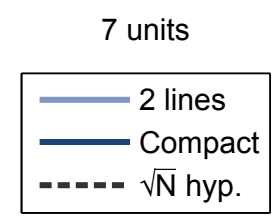

905

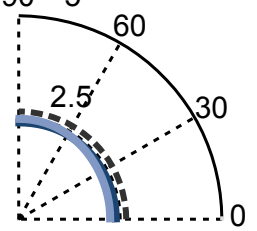

13 units

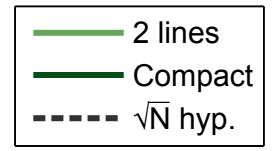

$90 \quad 5$

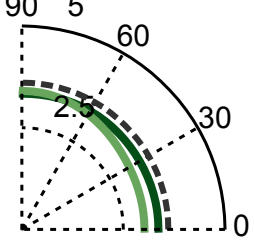

19 units

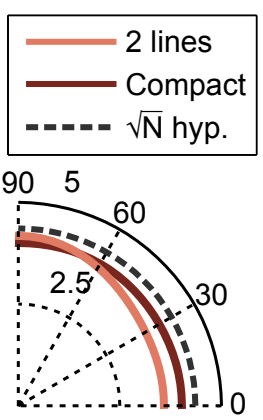

Figure 8: Farm-unit flicker ratio (ratio of the wave farm flicker severity to the unit flicker severity) as a function of the average wave direction (multidirectional waves) with 2 types of architecture and 3 different numbers of devices.

The results also show that the square-root hypothesis is realistic enough. In this condition, the flicker-production ratio shown in Fig. 5 would depend on the farm size and will be proportional to $N^{-1 / 2}$ (because average production is proportional to $N$ and flicker severity is proportional to $N^{+1 / 2}$ ). So, for grid integration, a good way to improve power quality is combine a large number of smaller devices in the farm. However, this may affect the total per-kWh cost of the WEC farm.

\section{Solutions and Compensation Actions}

All the solutions proposed here can have an impact on the per-kWh cost. In order to minimize this impact, the choice for different solutions must use life-cycle cost analysis in order to minimize the impact on the energy cost, as in [1].

\subsection{Verification of the Grid Code}

The individual limits given in Table 1 do not have the same significance in all cases; for example, the standard IEC [13] rec- ommends these values for users with a low agreed power. In other cases, the limits must be proportional to the cubic root of the agreed power.

For the British grid code[16], the limit given in Table 1 correspond to the loads that can be connected without further assessment. The authorized values corresponds to a total flicker severity at any point of the system of $P_{s t}=1.00$ and $P_{l t}=0.80$ for voltage below $132 \mathrm{kV}$. So the limit will depend on the other polluters around you.

For the French grid code[14], the limits are inversely proportional to the short-circuit apparent power if it is lower than $40 \mathrm{MVA}$ for Medium Voltage grid (rated voltage between $1 \mathrm{kV}$ and $50 \mathrm{kV}$ ). In other words, the polluter has less responsibility if the grid is too weak.

\subsection{Verification of the Interaction between the Wave Farm and the Grid}

If the grid is weak enough to cause flicker constraint problems, other grid constraints can also become difficult to respect, like maximum voltage deviation or thermal cable capacity. Indeed, a weak grid has very good chance to be linked with low energy consumption. Depending on the progress of the project, location and/or farm size could be change to avoid some issues. Otherwise the grid may have to be reinforced.

\subsection{Reactive Power Injection}

One way to limit voltage fluctuations is to consume reactive power proportional to the active power. In particular, according to (6) and Fig.3, voltage fluctuations are removed if the following relation is respected:

$$
Q_{\text {grid }}(t)=Q_{0}-P_{\text {grid }}(t) \cdot \tan (\Psi)^{-1}
$$

$Q_{0}$ is a constant value or a slow variable value (changing every hour) that can help to respect grid codes. It is considered negligible in the following, but constant injection or consumption of active or reactive power has no influence on flicker.

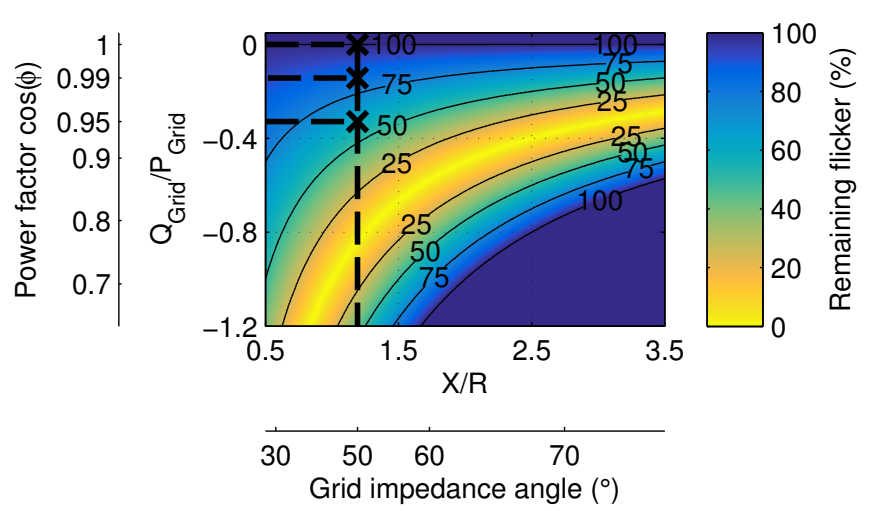

Figure 9: Ratio between the value of the flicker with and without reactive power consumption $(Q / P=0, P F=1)$ as a function of $\mathrm{X} / \mathrm{R}$ ratio and $\mathrm{Q} / \mathrm{P}$ ratio.

Fig.9 gives the ratio between the case with reactive power consumption (negative value with a generator convention) and 
Table 3: Solutions to inject negative reactive power

\begin{tabular}{llll}
\hline Location & Method & Advantages & Drawbacks \\
\hline \multirow{2}{*}{ At the PCC } & STATCOM & No need to filter harmonics & Cost \\
\cline { 2 - 4 } & TCR & Cost & Need to filter harmonics \\
\hline \multirow{2}{*}{ In each unit } & \multirow{2}{*}{ Use of grid side inverter } & No additional device & $\begin{array}{l}\text { More losses in submarine cables and in the in- } \\
\text { verter, Oversizing of the inverter }\end{array}$ \\
\hline
\end{tabular}

Table 4: Electrical Energy Storage Systems to smooth Wave Energy Converter production

\begin{tabular}{|c|c|c|}
\hline Location & Advantages & Drawbacks \\
\hline At the PCC & $\begin{array}{l}\text { Benefit from the scale effects Single module, Very } \\
\text { accessible Converter can be used as a STATCOM }\end{array}$ & High losses in all submarine cables \\
\hline At the substation & $\begin{array}{l}\text { Benefit from the scale effects, Relatively accessi- } \\
\text { ble, Fewer losses in the main submarine cable, Con- } \\
\text { verter can be used as a STATCOM }\end{array}$ & High losses in farm submarine cables \\
\hline In the DC-bus & $\begin{array}{l}\text { Redundancy, Fewer losses in all submarine cables, } \\
\text { No additional converter }\end{array}$ & Hardly accessible, Variable DC-bus voltage \\
\hline In each unit & $\begin{array}{l}\text { Redundancy, Fewer losses in all submarine cables, } \\
\text { Constant DC-bus voltage }\end{array}$ & Hardly accessible, Additional DC-DC converter \\
\hline
\end{tabular}

without reactive power consumption as a function of $\mathrm{X} / \mathrm{R}$ ratio and the $\mathrm{Q} / \mathrm{P}$ ratio.

For example, (12) or Fig. 9 can be used to find that, for a grid with an impedance angle of $50^{\circ}$, the flicker can be multiply by 0.83 with a power factor equal to 0.99 or multiply by 0.61 with a power factors equal to 0.95 .

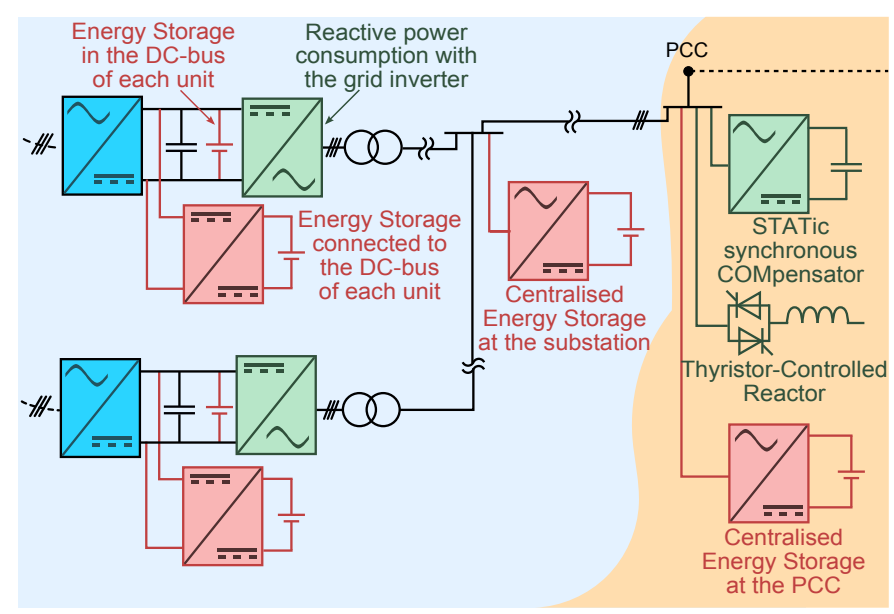

Figure 10: Different solutions to avoid Flicker issues in a Wave Energy Converters farm (two units and one substation represented): reactive power injection and energy storage systems.

Table 3 shows the different possibilities to inject reactive power at the PCC by using existing devices or by adding Flexible AC Transmission Systems (FACTS), like Static synchronous compensators (STATCOM) or Thyristor Controlled Reactors (TCR). In this last case, the current harmonic production due to TCR may have to be filtered in order to respect harmonic limitations [29]. Fig. 10 shows the placement of these devices for all the options presented.

\subsection{Change the Objective of the Control}

A possibility in order to limit flicker is to limit the power fluctuation by the control. Thus, a second objective can be added for powerful sea-state; limiting power fluctuations instead of just maximizing the energy converted. We can imagine in this case that a centralized control of each unit could give better results.

For the Direct Wave Energy Converters, one way can be to limit the absolute power. For OWC, the inertia of the turbine and the rotor can be used to smooth the power produced.

\subsection{Use of Electrical Energy Storage}

Electrical Energy Storage Systems (EESS) may also be used to smooth the power production.

Many storage technologies have been investigated in order to smooth wave energy production; supercapacitors[1, 30, 31], flywheels [32], Superconducting Magnetic Energy Storage (SMES) [33]. The smoothing time constant does not need to be very long (around $2 \mathrm{~s}$ ) in order to significantly reduce the flicker severity $[1,34]$.

The comparison for the placement of these EESS is described in Table 4. Fig. 10 shows the placement of these devices for all the options presented.

\section{Conclusion}

The flicker constraint, that limits voltage fluctuations, has been presented and the explanation why it could be a problem for Wave Energy Device grid integration has been given. An original representation has been presented: the intrinsic flicker, in order to give generic results in terms of grid characteristics.

The influences of the device type, its control and the seastate have been studied with a flicker-production ratio and have been compared. The influence of the architecture and the size of a farm on the flicker severity have been studied with a farmunit flicker ratio and have confirmed the classical square-root hypothesis when architecture is compact enough.

Finally, different solutions and compensatory actions are proposed in order to respect the flicker constraint and then allow grid integration of Wave Energy Converters farm. The best way 
to choose wisely the optimal combination of solutions seems to conduct Life Cycle Cost Analysis [1] in order to reduce the impact on per-kWh cost.

The simple model of the farm can be enhanced by taking into account WEC hydrodynamic interactions, mooring effects and better ways to take into account polychromatic and multidirectional behavior. Future studies can also use results from simulations, basin tests or sea testing trials in order to estimate flicker-production ratios and farm-unit flicker ratios in order to confirm results from this study.

\section{Acknowledgments}

This work has been supported by the French National Research Agency (ANR) within the project QUALIPHE (power quality and grid integration of direct wave energy converters), which is part of the PROGELEC program. This work was also supported by Science Foundation Ireland (SFI) under the Charles Parsons Award (Grant number 06/CP/E003) in collaboration with Marine Renewable Energy Ireland (MaREI), The SFI Centre for Marine Renewable Energy Research (12/RC/2302).

[1] T. Kovaltchouk, B. Multon, H. Ben Ahmed, J. Aubry, P. Venet, Enhanced aging model for supercapacitors taking into account power cycling: Application to the sizing of an Energy Storage System in a Direct Wave Energy Converter, in: Ecological Vehicles and Renewable Energies (EVER), Monte-Carlo, Monaco, 2014, pp. 1-10. doi:10.1109/EVER. 2014. 6844039.

[2] A. J. Nambiar, A. E. Kiprakis, A. R. Wallace, Quantification of voltage fluctuations caused by a wave farm connected to weak, rural electricity networks, in: International Conference on Harmonics and Quality of Power (ICHQP), Bergamo, Italy, 2010, pp. 1-8. doi : 10.1109/ICHQP. 2010.5625464.

[3] A. Blavette, D. L. O'Sullivan, R. Alcorn, T. W. Lewis, M. G. Egan, Impact of a Medium-Size Wave Farm on Grids of Different Strength Levels, Power Systems, IEEE Transactions on 29 (2) (2014) 917-923. doi:10.1109/TPWRS. 2013.2284513.

[4] E. Tedeschi, M. Santos-Mugica, Modeling and Control of a Wave Energy Farm Including Energy Storage for Power Quality Enhancement : the Bimep Case Study, Power Systems, IEEE Transactions on 29 (3) (2013) 1489-1497. doi:10.1109/TPWRS. 2013.2282213.

[5] S. Armstrong, D. Mollaghan, R. Alcorn, Effect of Wave Farm Aggregation on Power System Stability, in: Power Electronics for Distributed Generation Systems (PEDG), 2014 IEEE 5th International Symposium on, Galway, Ireland, 2014, pp. 1-6. doi :10.1109/PEDG . 2014. 6878699.

[6] A. Blavette, R. Alcorn, M. G. Egan, D. L. O’Sullivan, M. Machmoum, T. Lewis, A novel method for estimating the flicker level generated by a wave energy farm composed of devices operated in variable speed mode, in: Ecological Vehicles and Renewable Energies (EVER), Monte-Carlo, Monaco, 2014, pp. 1-5. doi:10.1109/EVER . 2014.6844041.

[7] F. Sharkey, J. MacEnri, E. Bannon, M. Conlon, K. Gaughan, Resourceinduced voltage flicker for wave energy converters - assessment tools, IET Renewable Power Generation 7 (6) (2013) 623-630. doi : 10 . 1049/ iet-rpg. 2012.0367.

[8] IEC 61400-21, Wind turbine generator systems - Measurement and assessment of power quality characteristics of grid connected wind turbines (2008).

[9] IEC 61000-4-15, Electromagnetic compatibility (EMC) - Part 4: Testing and measurement techniques - Section 15: Flickermeter - Functional and design specifications (2010).

[10] E. F. Fuchs, M. A. S. Masoum, Power Quality in Power Systems and Electrical Machines, Academic Press, 2011.

[11] P. Jourdan, Flickermeter Simulator (2009).

URL http://www.mathworks.fr/matlabcentral/ fileexchange/24423-flickermeter-simulator
[12] T. Kovaltchouk, K. Audoux, S. Rouland, J. Aubry, H. Ben Ahmed, B. Multon, Lissage dune production houlogénérée: gestion et dimensionnement dun système de stockage par supercondensateurs sous contrainte de flicker, in: Symposium de Génie électrique, Cachan, France, 2014, pp. $1-9$.

[13] IEC 61000-3-7, Assessment of emission limits for fluctuating loads in MV and HV power systems - Basic EMC publication, Tech. rep., International Electrotechnical Commission (IEC) (2008).

[14] F. decree, Arrêté of 23 April 2008 on the technical design and operation for the connection of a power generation facility to a public electricity network of distribution in low or medium voltage (in french), JORF 98.

[15] ESB Networks, Distribution Code, Tech. rep. (2014).

[16] System Utilisation Consultancy Group, Engineering Recommendation P28: Planning Limits for Voltage Fluctuations Caused by Industrial, Commercial and Domestic Equipment in the United Kingdom, in: Electricity Council Engineering Management Conference, 1989.

[17] Energinet.dk, Technical regulation 3.2 .5 for wind power plants with a power output greater than $11 \mathrm{~kW}$, Tech. rep., Energinet.dk (2010).

[18] T. Sun, Z. Chen, F. Blaabjerg, Flicker Study on Variable Speed Wind Turbines With Doubly Fed Induction Generators, Energy Conversion, IEEE Transactions on 20 (4) (2005) 896-905. doi:10.1109/TEC. 2005. 847993.

[19] M. Ammar, G. Joós, Impact of distributed wind generators reactive power behavior on flicker severity, Energy Conversion, IEEE Transactions on 28 (2) (2013) 425-433. doi : 10.1109/TEC. 2013. 2256425.

[20] L. Freris, D. Infield, Renewable energy in power systems, Wiley, 2008.

[21] D. P. Cashman, D. L. O'Sullivan, M. G. Egan, J. G. Hayes, Modelling and Analysis of an Offshore Oscillating Water Column Wave Energy Converter, in: European Wave and Tidal Energy Conference (EWTEC), Uppsala, Sweden, 2009, pp. 924-933.

[22] T. Kovaltchouk, B. Multon, H. Ben Ahmed, F. Rongère, A. Glumineau, J. Aubry, Influence of control strategy on the global efficiency of a Direct Wave Energy Converter with electric Power Take-Off, in: Ecological Vehicles and Renewable Energies (EVER), Monte-Carlo, Monaco, 2013, pp. 1-10. doi:10.1109/EVER. 2013.6521543.

[23] J. Aubry, H. Ben Ahmed, B. Multon, Sizing Optimization Methodology of a Surface Permanent Magnet Machine-Converter System over a Torque-Speed Operating Profile : Application to a Wave Energy Converter, Industrial Electronics, IEEE Transactions on 59 (5) (2012) 2116,2125. doi:10.1109/TIE. 2011.2163287.

[24] M. Molinas, O. Skjervheim, B. Sorby, P. Andreasen, S. Lundberg, T. Undeland, Power smoothing by aggregation of wave energy converters for minimizing electrical energy storage requirements, in: European Wave and Tidal Energy Conference (EWTEC), Porto, Portugal, 2007, pp. 3-8.

[25] A. Babarit, On the park effect in arrays of oscillating wave energy converters, Renewable Energy 58 (2013) 68-78. doi:10.1016/j .renene. 2013.03.008.

[26] T. Kovaltchouk, F. Rongère, M. Primot, J. Aubry, H. Ben Ahmed, B. Multon, Model Predictive Control of a Direct Wave Energy Converter Constrained by the Electrical Chain using an Energetic Approach, in: European Wave and Tidal Energy Conference (EWTEC), Nantes, 2015, pp. $1-10$.

[27] I. V. Lavrenov, Wind-Waves in Oceans: Dynamics and Numerical Simulation, Springer, 2003.

[28] R. F. Marsden, B. A. Juszko, Wind estimates from wave slopes, Journal of geophysical research 94 (C5) (1989) 6266-6272.

[29] IEC TR 61000-3-6, Electromagnetic compatibility (EMC) - Part 3-6: Limits - Assessment of emission limits for the connection of distorting installations to MV, HV and EHV power systems (2008).

[30] D. B. Murray, J. G. Hayes, D. L. O'Sullivan, M. G. Egan, Supercapacitor testing for power smoothing in a variable speed offshore Wave Energy Converter, Oceanic Engineering, IEEE Journal of 37 (2) (2012) 301-308.

[31] G. L. Park, A. I. Schäfer, B. S. Richards, Renewable energy-powered membrane technology: Supercapacitors for buffering resource fluctuations in a wind-powered membrane system for brackish water desalination, Renewable Energy 50 (2013) 126-135. doi:10.1016/j.renene. 2012.05.026.

[32] T. Yoshida, M. Sanada, S. Morimoto, Y. Inoue, Study of flywheel energy storage system for power leveling of wave power generation system, in: Electrical Machines and Systems (ICEMS), International conference on, Sapporo, Japan, 2012, pp. 1-5. 
[33] Z. Nie, X. Xiao, Q. Kang, R. Aggarwal, H. Zhang, W. Yuan, SMESBattery Energy Storage System for Conditioning Outputs From Direct Drive Linear Wave Energy Converters, Applied Superconductivity, IEEE Transactions on 23 (3) (2013) 1-5. doi:10.1109/TASC. 2013. 2246852.

[34] A. Blavette, D. L. O'Sullivan, T. W. Lewis, M. G. Egan, Dimensioning the equipment of a wave farm: Energy storage and cables, in: Ecological Vehicles and Renewable Energies (EVER), Monte-Carlo, Monaco, 2013, pp. 1-9. doi:10.1109/EVER.2013.6521587. 